\title{
Are Only Anatomic Outcomes Important After Pelvic Organ Prolapse Surgeries?
}

\author{
Mertihan Kurdoglu*
}

$\mathrm{O}$ ver the next few years, it is anticipated that the number of operations for pelvic organ prolapse (POP) and related pelvic floor disorders will increase substantially as a result of aging of the population (1). Nowadays, roughly, 1 out of every 10 women undergoes surgery for urinary incontinence or prolapse. When we also consider $30 \%$ risk of reoperation during their lifetime, it is obvious that surgical treatment of POP has an important impact on a women's health (2).

Anatomic outcomes and relief of specific prolapse symptoms following any POP surgery are focus of current studies on this field. However, the women with POP often present with not only the usual complaints of vaginal pressure or bulging, but also pelvic pain and the other pelvic symptoms related to the lower urinary tract, bowel, and sexual function $(3,4)$. Therefore, the results related to the improvement in quality of life and correction of these preoperative associated pelvic symptoms are also important. In addition, de novo urinary and defecatory symptoms appearing as new pelvic symptoms after surgery for prolapse and incontinence can be as high as $22 \%$ to $27 \%$ and they should also be reported (3).

With the advance of technology and medical knowledge, in addition to several classical abdominal and vaginal procedures for the restoration of POP, their minimally invasive versions such as laparoscopic or robot-assisted laparoscopic surgeries are becoming more popular. The data on these newer surgical procedures also lack robust information related to their functional outcomes (3). Therefore, short, and long-term outcomes related to the changes in existing or the appearance of new lower urinary tract symptoms, pelvic pain, and bowel functions following not only classical but also these new techniques should be presented with comparative studies.

As a result, a comprehensive preoperative counselling not only on anatomic outcomes but also on the functional outcomes of the surgery, including the rate of improvement and the risk of development of de novo lower urinary tract,

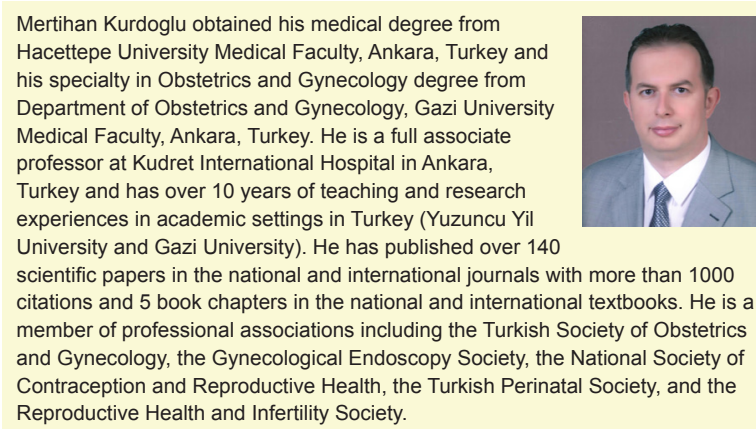

scientific papers in the national and international journals with more than 1000 citations and 5 book chapters in the national and international textbooks. He is a member of professional associations including the Turkish Society of Obstetrics and Gynecology, the Gynecological Endoscopy Society, the National Society of Contraception and Reproductive Health, the Turkish Perinatal Society, and the Reproductive Health and Infertility Society.

defecatory, and sexual symptoms should be given to all patients planning to undergo a POP surgery with a specific technique.

\section{Ethical Issues}

Not applicable.

\section{Conflict of Interests}

The author has no conflicts of interest to disclose.

\section{References}

1. Wu JM, Kawasaki A, Hundley AF, Dieter AA, Myers ER, Sung VW. Predicting the number of women who will undergo incontinence and prolapse surgery, 2010 to 2050. Am J Obstet Gynecol. 2011;205:230e1-5. doi: 10.1016/j.ajog.2011.03.046

2. Olsen AL, Smith VJ, Bergstrom JO, Colling JC, Clark AL. Epidemiology of surgically managed pelvic organ prolapse and urinary incontinence. Obstet Gynecol 1997;89:501-6. doi: 10.1016/S0029-7844(97)00058-6

3. Segal S, Arya LA, Smith AL. Functional Outcomes for Incontinence and Prolapse Surgery. Curr Bladder Dysfunct Rep 2012;7: 179-86. doi: 10.1007/s11884-012-0136-9

4. Weber AM, Abrams P, Brubaker L, Cundiff G, Davis G, Dmochowski RR, et al. The standardization of terminology for researchers in female pelvic floor disorders. Int Urogynecol J Pelvic Floor Dysfunct. 2001;12:178-86.

\footnotetext{
Copyright ( 2018 The Author(s); This is an open-access article distributed under the terms of the Creative Commons Attribution License (http://creativecommons.org/licenses/by/4.0), which permits unrestricted use, distribution, and reproduction in any medium, provided the original work is properly cited.
} 